\title{
ESSENTIAL COMPLETIONS OF DISTRIBUTIVE LATTICES
}

\author{
Gerhard Gierz and Albert R. Stralka
}

\begin{abstract}
The salient feature of the essential completion process is that for most common distributive lattices it will yield a completely distributive lattice. In this note it is shown that for those distributive lattices which have at least one completely distributive essential extension the essential completion is minimal among the completions by infinitely distributive lattices. Thus in its setting the essential completion of a distributive lattice behaves in much the same way as the one-point compactification of a locally compact topological space does in its setting.
\end{abstract}

The purpose of this paper is to study a completion process for distributive lattices suggested by the procedure used by B.Banascheswki and G. Bruns in [2] to construct injective hulls for distributive lattices. As will be seen it is not inappropriate to call this completion the essential completion.

To construct the injective hull, or, what is the same thing, the maximal essential extension of a distributive lattice $L$, Banaschewski and Bruns first imbed $L$ into an arbitrary Boolean algebra $B^{\prime}$, next take $B$ to be the Boolean subalgebra of $B^{\prime}$ generated by $L$, and finally take

Received 26 March 1985.

Copyright Clearance Centre, Inc. Serial-fee code: 0004-9727/85 $\$ A 2.00+0.00$. 
$B(L)$ to be the Dedekind - MacNeille completion of $B$. It turns out that $B(L)$ is uniquely determined by $L$ and the canonical imbedding of $L$ into $B(L)$ is essential in the sense that non-trivial congruence relations on $B(L)$ have non-trivial restrictions to (the image of) $L$. Since complete Boolean algebras do not allow proper essential extensions, it follows that $B(L)$ is the maximal essential extension of $L$. It is not $B(L)$ itself that directly concerns us but $\rho(L)$ the smallest complete sublattice of $B(L)$ containing $L$. Since $\rho(L)$ is an essential extension of $L$ we call it the essential completion of $L$.

A distributive lattice which has at least one completely distributive essential extension is said to be essentially completely distributive. This property is closely related to separation properties of the Zariski topology. It turns out that the essential completion of a distributive lattice is completely distributive precisely in those cases in which the Zariski topoloty is Hausdorff (see [1] and [6]). Most common distributive lattices have this property (cf [6]).

our main result is that the essential completion functions in its setting behaves in much the same way as the one-point compactification does in the context of locally compact Hausdorff spaces. Specifically, we prove: Let $L$ an essentially completely distributive lattice. Then there is a completely distributive lattice $p(L)$ and a topologically and algebraically dense imbedding $i: L \rightarrow \rho(L)$ such that whenever $f: L \rightarrow M$ is a dense lattice imbedding of $L$ into an infinitely distributive lattice $M$ then there is a unique complete lattice homomorphism $g: M \rightarrow p(L)$ such that $g \circ f=i$. Moreover, the lattice $p(L)$ is uniquely determined by these properties.

For chains the essential completion and the Dedekind-MacNeille completion coincide. However as we saw above the essential completion remains in the category of distributive lattices while it is well-known that the Dedekind-MacNeille completion does not always do so. These completions also differ in that the Dedekind-MacNeille completion provides a lattice which is only complete whereas the essential completion, at least in most common cases, provides a completely distributive completion. We conclude the paper by characterizing $\rho(L)$ in terms of ideals and filters thereby providing a somewhat clearer picture of this difficult object. 


\section{Preliminaries}

For the basic facts and terminology on lattices and topological lattices we refer to [3] and [4].

Recall that the Zariski topology on a distributive lattice has as a subbase for its closed sets all sets of the form

$$
\begin{aligned}
& {[a \wedge x \leq b]=\{x \in L: a \wedge x \leq b\}} \\
& {[a \vee x \geq b]=\{x \in L: a \vee x \geq b\}}
\end{aligned}
$$

where $a$ and $b$ range over the elements of $L$ (see [6]). When we refer to a topological property on a distributive lattice and indicate no specific topology, then we are referring to the Zariski topology.

A distributive lattice $L$ is meet-continuous if for any subset $A \subset L$ and any element $b \in L$ the following equality holds:

$$
b \wedge(\sup A)=\operatorname{sip}\{b \wedge a: a \in A\} \text {. }
$$

Join-continuity is defined dually. A distributive lattice which is both meet-continuous and join-continuous is said to be infinitely distributive.

The sublattice $L$ of the complete lattice $M$ is algebraically dense in $M$ if $M$ is the smallest complete sublattice of $M$ containing L.

Our work depends upon the realization of $B(L)$ which we developed in [5] enabling us to keep discussion restricted to congruence lattices. For a distributive lattice $L$ let $\Theta(L)$ be its congruence lattice with $\Delta$ the identity congruence or diagonal, and $\nabla$ the congruence which collapses the whole lattice $L$ to a single point. The congruence lattice of $L$ is a meet-continuous lattice. We can identify $B(L)$ with the subset of all pseudocomplements in $\Theta(L)$ endowed with the inherited order from $\theta(L)$ but not necessarily the inherited operations. We define the imbedding $i: L \rightarrow B(L)$ by $i(x)=\theta_{x}=\{(a, b) \in L \times L: a \vee x=b \vee x\}$.

The pseudocomplement of $\theta_{x}$ is given by $\theta_{x}^{\perp}=\theta^{x}=\{(a, b) \epsilon L \times L$ : $x \wedge a=x \wedge b\} . \theta^{x}$ is the complement of $\theta_{x}$ in $B(L)$.

Within $B(L)$ we take $\rho(L)$, the essential completion of $L$, to be the smallest complete sublattice containing $i(L)$. It was shown in [5] and [6] that $i$ is an essential imbedding into both $\rho(L)$ and $B(L)$. If $L$ is an essentially completely distributive lattice, then $\rho(L)$ is 
completely distributive (see [1] and [6]). Moreover, in this case if both $L$ and $\rho(L)$ are equipped with that topology, then $i$ is a topological imbedding.

\section{The Main Result}

THEOREM 2.1. Let $L$ be a distributive lattice cond assione that $L$ is an essentially completely distributive lattice. Then there is a completely distributive lattice $\rho(L)$ and a (topological and algebraical) dense imbedding $i: L \rightarrow \rho(L)$ such that whenever $f: L \rightarrow M$ is a lattice imbedding into an infinitely distributive lattice $M$ such that $f(L)$ is algebraically dense in $M$, then there is a unique complete lattice homomorphism $g: M \rightarrow \rho(L)$ satisfying $g \circ f=i$. Moreover, the lattice $\rho(L)$ is uniquely determined by these properties.

Remark. Since the imbedding of $L$ into $\rho(L)$ is topological and since $\rho(L)$ is a compact distributive lattice, the lattice $\rho(L)$ may be viewed as the minimal compactification of $L$ in the Zariski topology.

The proof of (2.1) will be done in a series of propositions and lemmas.

Throughout this section we shall assume that $L$ is a distributive lattice which is essentially completely distributive, that is, which is Hausdorff in the Zariski topology.

PROPOSITION 2.2. If $A$ and $B$ are subsets of $L$, then inf $i(A) \leq \sup i(B)$ if and only if $\Delta=n\left\{\theta_{x} \cap \theta^{\mathcal{Y}}: x \in A, y \in B\right\}$.

Proof. We have inf $i(A) \leq \sup i(B)$ if and only if $i(A) \cap(\sup i(B))^{\perp}=\Delta$. Thus, the claim follows from

$$
\begin{aligned}
(\sup i(B))^{\perp} & =\inf \left\{i(y)^{\perp}: y \in B\right\} \\
& =\inf \left\{\theta_{y}^{\perp}: y \in B\right\} \\
& =\inf \left\{\theta^{y}: y \in B\right\}
\end{aligned}
$$

PROPOSITION 2.3. If $A$ and $B$ are subsets of $L$ and if inf $f(A) \leq \sup f(B)$, then inf $i(A) \leq \operatorname{sip} i(B)$.

Proof. Assume the inequality $\inf i(A) \leq \sup i(B)$ is not true. Then applying Proposition (2.2) we can find a pair of distinct elements 
$(a, b) \in L^{2}$ which belongs to $\theta_{x}$ for all $x \in A$ and to $\theta^{y}$ for all $y \in B$. Hence we have

$$
\begin{array}{ll}
a \vee x=b \vee x & \text { if } x \in A, \\
a \wedge y=b \wedge y & \text { if } y \in B .
\end{array}
$$

Now let $l=\inf f(A)$ and let $r=\sup f(B)$. since $f$ is a lattice imbedding, $f(a)$ and $f(b)$ are distinct. Moreover, since $M$ is infinitely distributive,

$$
\begin{aligned}
& f(a) \vee z=f(b) \vee z, \\
& f(a) \wedge r=f(b) \wedge r .
\end{aligned}
$$

Since by assumption $\tau \leq r$, the second of these two equations implies that

$$
\begin{aligned}
f(a) \wedge Z & =f(a) \wedge r \wedge Z \\
& =f(b) \wedge r \wedge Z \\
& =f(b) \wedge z .
\end{aligned}
$$

Thus both $f(a)$ and $f(b)$ are relative complements of $l$. In a distributive lattice relative complements are unique, so we may conclude that $f(a)=f(b)$, contradicting the injectivity of $f$.

For a distributive lattice $T$, Spec $T$ will denote the set of all prime elements of $T$ and Cospec $T$ will be the set of coprime elements (cf [4]). We write $x \ll y$ to denote that $x$ is way below $y$, that is if $V$ is an upward directed set with $\sup V \geq y$, then there is $v \in V$ such that $x \leq v$. The statement that $x$ is way above $y$ is denoted by $x \gg y$ and is defined dually. Note that $x \ll y$ and $y \gg x$ are not equivalent.

If $T$ is a completely distributive lattice, then from [7] we know:

(*) If $p \in \operatorname{Spec} T$, then the set $\{q: q \gg p, q \in$ Spec $T\}$ is down directed and has infimum $p$.

(**) (Interpolation Property) Whenever $p, q \in$ Spec $T$ are primes such that $q \gg p$, then there is a prime element $r \in$ Spec $T$ such that $q \gg r \gg p$.

$(* \star \star)$ Every element of $T$ is an infimum of prime elements and a supremum of coprime elements (that is Spec $T$ order generates $T$ and Cospec $T$ order generates $T^{O p}$, the opposite of $T$ ).

Now suppose that $M$ is an infinitely distributive lattice and $f: L \rightarrow M$ is a dense lattice imbedding. Instead of defining the mapping $g: M \rightarrow \rho(L)$ directly, we will construct the upper and lower adjoint 
of $g$. We begin by defining a map from spec $\rho(L)$ to $M$ by $\phi_{0}:$ Spec $\rho(L) \rightarrow M$

$$
\rho \rightarrow \operatorname{in} f\{\sup \{f(x): i(x) \leq p, x \in L\}: q \gg p, q \in \operatorname{Spec} \rho(L)\} .
$$

Then $\phi_{0}$ preserves directed infima: Indeed, let $A \subset \rho(L)$ be a downward directed set of primes and assume that $p=\inf A$. We have to show that

$$
\phi_{0}(p)=\inf \phi_{0}(A) \text {. }
$$

Clearly, since $\phi_{0}$ is monotone, we have $\phi_{0}(p) \leq$ inf $\phi_{0}(A)$. In order to verify the other inequality, we will prove

(C) For every $q \in$ Spec $\rho(L)$ with $q \gg p$ there is an element $r \in A$ such that $\sup \{f(x): i(x) \leq q, x \in L\} \geq \phi_{0}(r)$.

Indeed, pick $r \in A$ such that $q>r \geq p$, which is possible because of the interpolation property for $\gg$. Then we have

$$
\begin{aligned}
\sup \{f(x): i(x) \leq q, x \in L\} & \geq \inf \left\{\sup \left\{f(x): i(x) \leq q^{\prime}, x \in L\right\}:\right. \\
& \left.q^{\prime}>>, q^{\prime} \in \operatorname{spec} p(L)\right\} \\
& =\phi_{0}(r) .
\end{aligned}
$$

Now from (C) we conclude that

$$
\begin{aligned}
\phi_{0}(p) & =\inf \{\sup \{f(x): i(x) \leq q, x \in L\}: q>p, q \in \text { Spec } \rho(L)\} \\
& \geq \inf \left\{\phi_{0}(r): r \in A\right\} \\
& =\inf \phi_{0}(A) .
\end{aligned}
$$

In the next step, we define a map

$$
\begin{aligned}
\phi: \rho(L) & \rightarrow M \\
u & \rightarrow \inf \left\{\phi_{0}(p): u \leq p, p \in \operatorname{spec} p(L)\right\} .
\end{aligned}
$$

obviously, $\phi$ is monotone. To see that $\phi$ preserves finite infima, let $u$ and $v$ be two arbitrary elements of $\rho(L)$. Then the monotonicity of $\phi$ implies that $\phi(u \wedge v) \leq \phi(u) \wedge \phi(v)$. Conversely, let $p$ be a prime element above the infimum of $u$ and $v$. Then $p$ is actually above either $u$ or $v$, yielding $\left\{\phi_{0}(p): u \wedge v \leq p, p \in \operatorname{Spec} \rho(L)\right\}=\left\{\phi_{0}(p)\right.$ : $u \leq p, p \in \operatorname{spec} p(L)\} \cup\left\{\phi_{0}(p): v \leq p, p \in \operatorname{Spec} \rho(L)\right\}$. Thus, $\phi(u \wedge v) \geq \phi(u) \wedge \phi(v)$. 
Our next claim is that $\phi$ preserves down directed infima. Let $A$ be a down directed subset of $\rho(L)$. The inequality $\phi(\inf A) \leq \inf \phi(A)$ is again obvious. Conversely, let $p, q \in \operatorname{Spec\rho }(L)$ such that $A \leq p$ and $q \gg p$. By the interpolation property we may pick a prime element $p^{\prime}$ such that $q \gg p^{\prime} \gg p$. Since $A$ is down directed and has an infimum less than or equal to $p$, there is an element $a \in A$ such that $a \leq p^{\prime}$. We obtain

$$
\text { inf } \begin{aligned}
(A) & \leq \phi(a) \\
& \leq \phi_{0}\left(p^{\prime}\right) \\
& \leq \phi_{0}(q) .
\end{aligned}
$$

Since $q \gg p$ was arbitrary, since $\phi_{0}$ preserves infima of down directed sets of primes and since $\{q \in$ Spec $\rho(L): q>p\}$ is down directed with infimum $p$, we conclude that

$$
\inf \phi(A) \leq \phi_{0}(p) .
$$

Finally, since $p$ was an arbitrary prime element such that inf $A \leq p$, we have

$$
\inf \phi(A) \leq \phi(\inf A)
$$

from the definition of $\phi$.

We conclude

PROPOSITION 2.4. The mopping $\phi: \rho(L) \rightarrow M$ preserves arbitrary infima.

$$
\begin{aligned}
& \text { Dually, we define maps } \psi_{0}: \text { Cospec } \rho(L) \rightarrow M \\
& \qquad c \rightarrow \sup \{i n f\{f(x): d \leq i(x), x \in L\}: c \ll d, d \in \operatorname{Cospec} \rho(L)\}
\end{aligned}
$$

and

$$
\begin{aligned}
\psi: \rho(L) & \rightarrow M \\
u & \rightarrow \sup \left\{\phi_{0}(c): c \leq u, c \in \text { Cospec } \rho(L)\right\} .
\end{aligned}
$$

PROPOSITION 2.5. The mopping $\psi: \rho(L) \rightarrow M$ preserves arbitrary suprema.

We will now show that the mappings $\phi$ and $\psi$ are the upper and lower adjoint of the mapping $g: M+\rho(L)$.

PROPOSITION 2.6. For $a, b \in \rho(L), \psi(a) \leq \phi(b)$ if and only if $a \leq b$.

Proof. First, let us assume that $a \leq b$. We have to show: If $c$ is a coprime and if $p$ is a prime such that $c \leq a \leq b \leq p$, then 
$\psi_{0}(c) \leq \phi_{0}(p)$. This is the same as showing that if $d \in \operatorname{Cospec} p(L)$, $d \ll c \leq a$ and if $q \in \operatorname{Spec} p(L), q>p \geq b$ then

$$
\inf \{f(x): d \leq i(x), x \in L\} \leq \sup \{f(y): i(y) \leq q, y \in L\} .
$$

Note that the set $\{z \in \rho(L): d<z$ and $q \gg z\}$ is a neighbourhood of the elements $a, b \in \rho(L)$ (this is an consequence of (I.2.5, p. 59), (II.1.10, p. 104), (II.1.6, p. 144), (VIII.2.8, p. 318) and (VII.2.9, p. $318)$ of [4]). Since $i(L)$ is dense in $\rho(L)$, this set contains an element of the form $i\left(x^{\prime}\right), x^{\prime} \in L$. Hence there is an element $x^{\prime} \in L$ such that $d \leq i\left(x^{\prime}\right) \leq q$. This yields

$$
\begin{aligned}
\inf f f(x): d \leq i(x), x \in L\} & \leq f\left(x^{\prime}\right) \\
& \leq \operatorname{sip}\{f(y): i(y) \leq q, y \in L\},
\end{aligned}
$$

as desired.

Now assume that we are given two elements $a, b \in \rho(L)$ such that

$a \leqslant b$. Since primes and coprimes both order generate the lattice $\rho(L)$

(see $(* \star \star))$ ), we can find primes $p, q$ and coprimes $c, d$ such that

$$
\begin{aligned}
d \ll c & \leq a, \\
q \gg p & \geq b, \\
d & \leq q .
\end{aligned}
$$

If we can show that the inequality

$$
\inf \{f(x): d \leq i(x), x \in L\} \leq \sup \{f(y): i(y) \leq q, y \in L\}
$$

cannot hold we would be certain that $\psi_{0}(c) \& \phi_{0}(p)$ and hence $\psi(a) \& \phi(b)$. Thus, let us assume that the above inequality is true. Then Proposition (2.3) would yield

$$
\begin{aligned}
d & \leq i n f\{i(x): d \leq i(x), x \in L\} \\
& \leq \sup \{i(y): i(y) \leq q, y \in L\} \\
& \leq q
\end{aligned}
$$

contradicting the choice of $d$ and $q$.

PROPOSITION 2.7. For every $x \in L$ we have $\psi(i(x)) \leq f(x) \leq \phi(i(x))$.

Proof. For every $x \in L$ we have

$$
\phi(i(x))=\inf \left\{\phi_{0}(p): i(x) \leq p, p \in \operatorname{spec} \rho(L)\right\} \text {. }
$$

Hence, in order to verify $f(x) \leq \phi(i(x))$ we must show that $f(x) \leq \phi_{0}(p)$ whenever $i(x) \leq p, p \in$ Spec $p(L)$. By the definition of $\phi_{0}$ this is equivalent to 


$$
f(x) \leq \operatorname{sip}\{f(y): i(y) \leq q, y \in L\}
$$

whenever $q \gg p, q \in$ Spec L. But if $q \gg p$, then $i(x) \leq p \leq q$, hence $f(x) \in\{f(y): i(y) \leq q, y \in L\}$ and therefore the above inequality holds trivially.

Similarly, $\psi(i(x)) \leq f(x)$ for every $x \in L$.

Let $g_{0}$ be the lower adjoint of $\phi$, and $g_{1}$ the upp.sr adjoint of

$\psi$. Then (2.7) implies

PROPOSITION 2.8. $g_{0} f(x) \leq i(x) \leq g_{1} f(x)$.

PROPOSITION 2.9. The Zower adjoint $g_{0}$ of $\phi$ agrees with the upper adjoint $g_{1}$ of $\psi$. Especially, if we define $g=g_{0}=g_{1}$, then $g$ is a complete lattice homomorphism, and $g \circ f=i$.

Proof. For any $u \in M$ we have $\psi g_{1}(u) \leq u \leq \phi g_{0}(u)$ and so $g_{1}(u) \leq g_{0}(u)$ by $(2.6)$. Moreover, the set $\left\{u \in M: g_{0}(u) \leq g_{1}(u)\right\}$ is a complete sublattice of $M$ since $g_{0}$ preserves suprema and $g_{1}$ preserves infima. But for $x \in L, g_{0} f(x) \leq i(x) \leq g_{1}(x)$ by (2.8), so this set contains the image of $f$ and hence is the whole of $M$. This proves $g_{0}=g_{1}$. The equality $g \circ f=i$ now follows from (2.8).

This last proposition finishes the major part of the proof of (2.1). Note that the uniqueness of $g$ follows from the fact that $f(L)$ is algebraically dense in $M$. The fact that $\rho(L)$ is uniquely determined by all these properties follows from general category theory.

It is now appropriate to list some consequences of Theorem (2.1) and the fact that in our discussion we needed the map $f: L \rightarrow M$ to be a lattice homomorphism only in the proof of Proposition (2.3). Note that (2.3) is trivial in the case that $L$ itself is completely distributive and hence agrees with $\rho(L)$ :

COROLLARY 2.10. Let $L$ be a completely distributive lattice densely imbedded (as a partially ordered set) into a infinitely distributive $M$. Then $L$ is a retract of $M$ under a complete lattice homomorphism.

COROLLARY 2.11. Let $M$ be a compact distributive lattice. If $M$ contains a dense essentially completely distributive subtattice, then $M$ admits non-trivial continuous lattice homomorphisms into the unit interval. 
COROLLARY 2.12. Let $M$ be a compact distributive lattice. If $M$ contains an order isomorphic copy of a completely distributive lattice which is dense in $M$, then $M$ admits non-trivial continuous lattice homomorphism into the unit interval.

It turns out that completely distributive lattices and essentially completely distributive lattices are also characterized by all those properties listed in the corollaries:

THEOREM 2.13. If $L$ is a lattice such that for every infinitely distributive Zattice $M$ and every dense imbedding $i: L \rightarrow M$ there is a complete lattice homomorphism $f: M \rightarrow L$ such that $f \circ i=i d_{L}$, then $L$ is completely distributive.

The proof of this theorem follows from the fact that every distributive lattice admits a dense imbedding into a completely distributive lattice and that every quotient of a completely distributive lattice under a complete lattice homomorphism is again completely distributive.

We will now show that $(2.1)$ is best possible in the sense that we cannot weaken the assumption that $L$ be essentially completely distrivutive: Assume that we are given a lattice $L$ together with a dense embedding $e: L \rightarrow L^{\prime}$, where $L^{\prime}$ is an infinitely distributive lattice such that for every other dense imbedding $f: L \rightarrow M$ into a infinitely distributive lattice there is a complete lattice homomorphism $g: M \rightarrow L^{\prime}$ satisfying $g \circ f=e$. since $f$ is a dense imbedding, the mapping $g$ must be uniquely determined. Let $\rho(L)$ be the "closure" of $L$ in the maximal essential extension $B(L)$ of $L$ (that is $\rho(L)$ is again the smallest complete sublattice of $B(L)$ containing the image of $i(L)$ ). Then $L$ is densely imbedded in $\rho(L)$, and, although $\rho(L)$ need not be completely distributive, it will still be infinitely distributive. (Recall that every complete Boolean algebra and hence every complete sublat tice of a complete Boolean algebra is infinitely distributive). It follows that there is a complete lattice homomorphism $g: \rho(L) \rightarrow L^{\prime}$. This mapping $g$ has to be surjective; it is also injective since the composition with the essential imbedding $i: L \rightarrow \rho(L)$ yields the imbedding $e$. This argument shows that $\rho(L)$ is the only possible choice for proving a result like Theorem (2.1). A similar argument to the one used in the proof of Theorem (2.13) yields 
THEOREM 2.14. Let $L$ be a distributive lattice and suppose that $L$ adrits a dense imbedding $e: " L \rightarrow L^{\prime}$ into an infinitely distributive lattice $L^{\prime}$ such that whenever $f: L \rightarrow M$ is a second such dense imbedding into an infinitely distributive lattice $M$, then there is a (uniquely determined) complete lattice homomorphism $g: M \rightarrow L^{\prime}$ such that $g \circ f=e$. Then, $u p$ to a cononical isomorphism, $L^{\prime}=\rho(L)$ and $e=i$. Moreover, in this case $\rho(L)$ is completely distributive and therefore $L$ is essentially completely distributive.

3. A Characterization of $\rho(L)$ by Means of Closed Filters and Ideals.

In this section we give a description of $\rho(L)$ based upon the Zariski topology of $L$. Recall that the imbedding of $L$ into $\rho(L)$ is a topological imbedding for the Zariski topology. This fact will play a prominent role in our discussion.

The next result follows from Propositions (4.2), (4.3), and $[6,4.4]$.

PROPOSITION 3.1. Let $L$ be a distributive lattice and let $I \subset L$ be con ideal of $L$. Then the following statements are equivalent:

(1) I is closed.

(2) If $D \in I$ is a directed subset and if $D$ converges to an element $x \in L$ in the 2ariski topology, then $x \in I$.

(3) If $D \subset I$ is a directed subset such that sup $D$ exists in $L$ and such that $a \wedge \sup D=\sup d \in D^{(d \wedge a)}$ for every $a \in L$, then $\sup D \in I$.

(4) If $x \in L$ has the property that $x \wedge a=\sup (I \cap t(x \wedge a))$ for every $a \in L$, then $x \in I$.

From now on, we will identify $L$ with the sublattice $i(L)$ of $\rho(L)$.

PROPOSITION 3.2. If $I$ and $J$ are two different closed ideals of $L$, then $I$ and $J$ have different suprema in $\rho(L)$.

Proof. Assume that sup $I=$ sup $J$. Take $j$ to be an arbitrary element of $J$. Then $\sup _{\rho(L)^{I}}$ is an upper bound of $j$ in $\rho(L)$. Since $\rho(L)$ is infinitely distributive, we conclude that $j=\sup _{\rho}(L)^{(I \cap+j)}$. Moreover, since the Zariski topology on $\rho(L)$ is the interval topology, 
the directed set $I n \downarrow j$ converges to $j$ in the zariski topology on $\rho(L)$. The imbedding of $L$ into $\rho(L)$ is topological, therefore $I n \downarrow j$ also converges to $j$ in the Zariski topology of $L$. Hence, by property (2) of Proposition (3.1), $j$ belongs to $I$. This yields $J \subset I$. By symmetry, $I=J$.

Let $A$ be a subset of a complete lattice. We define

$$
\begin{aligned}
& A^{+}=\{\sup D: D \text { is a up-directed subset of } A\}, \\
& A^{-}=\{\sup F: F \text { is a down-directed subset of } A\} .
\end{aligned}
$$

Further, if $L$ is an arbitrary distributive lattice, we let

$$
\begin{aligned}
& I_{c}(L)=\{I \subset L: I \text { is a closed ideal of } L\}, \\
& F_{c}(L)=\{F \subset L: F \text { is a closed filter of } L\} .
\end{aligned}
$$

When ordered by inclusion, $I_{c}(L)$ and $F_{c}(L)$ are complete lattices.

The infimum in these lattices agrees with set theoretical intersection. However, it is not in general true that the supremurn of two closed ideals taken in the lattice $I_{c}(L)$ is the same as the supremum of those two ideals taken in the lattice of all ideals (see the following exampled. Therefore, it is not obvious that the lattice of all closed ideals (filters) is again distributive (see Theorem (3.4)).

EXAMPLE 3.3. Let $L$ be the open wonit square enriched by appending the point (1.1). Then the zariski topology on $L$ is the topology induced by the Euclidean topology of the plane. Let

$$
\begin{aligned}
& I=\left\{(x, y) \in L: y \leq \frac{1}{2}\right\}, \\
& J=\left\{(x, y) \in L: x \leq \frac{1}{2}\right\} .
\end{aligned}
$$

Then the supremum of $I$ and $J$ in the ideal lattice of $L$ contains all the elements of $L$ except the point (1.1) and hence is not closed.

In the following result, $L$ will again be identified with a subset of $\rho(L)$. Hence $L^{+}$must be evaluated in the lattice $\rho(L)$ and not in $L$. Even when $L$ is a complete lattice, $L^{+}$does not have to agree with $L$. Note that the meet-continuity of $\rho(L)$ implies that $L^{+}$is a sublattice of $\rho(L)$. Therefore, $L^{+}$will always be a distributive lattice. 
THEOREM 3.4. The lattice $L^{+}$is isomorphic with $I_{c}(L)$; an isomorphism between $I_{c}(L)$ and $L^{+}$is given by the mopping

$$
I \leftrightarrow \operatorname{sip} I \text {. }
$$

Its inverse is the mopping

$$
x \rightarrow\{y \in t: y \leq x\} .
$$

Thus, $I_{c}(L)$ is a distributive lattice.

Proof. By Proposition (3.2), the mapping $I \rightarrow s u p I$ is injective. If $x \in L^{+}$, then $x$ is a directed supremum (and hence the supremum of an ideal) of elements of $L$. Thus, $x$ has to be the supremurn of the closed ideal $\{y \in L: y \leq x\}=\downarrow x \cap L$. Since there is no more than one closed ideal with supremum $x$, the mapping $x \rightarrow\{y \in L: y \leq x\}$ is the inverse of $I \rightarrow \sup I$.

When $L$ is a complete meet-continuous lattice, an ideal of $L$ is closed if and only if it is a lower set of a point. In this case $L$ and $I_{c}(L)$ will be isomorphic and therefore $I_{c}(L)$ will be meet-continuous too. This last property remains true in general:

PROPOSITION 3.7. If $L$ is any distrivutive zattice, then $I_{c}(L)$ is meet-continuous.

Proof. This follows easily from the fact that $I_{c}(L)$ is isomorphic with $L^{+}$and that $L^{+}$is closed under finite infima and arbitrary suprema in the complete Boolean algebra $B(L)$.

Now let us examine the join-continuity of $I_{c}(L)$. Unfortunately, the imbedding $I \leftrightarrow$ sup $I$ does not preserve arbitrary infima and in general, $I_{c}(L)$ need not be join-continuous. (Consult the open unit square for examples: If $L$ is the open unit square, then $I_{c}(L)=L^{+}=$ $\left\{(x, y) \in B^{2}: 0<x, y \leq 1\right\}$ and this lattice is not join-continuous. $)$ However, what happens if $L$ is a complete join-continuous lattice to begin with? Is it then true that $I_{c}(L)$ is meet-continuous and joincontinuous? If the answer to this quention were positive, then $\rho(L)$ would always be of the form $\rho(L)=L^{+}=L^{-+}$. We will leave the general case as an open problem and only discuss a special case: 
If $L$ is essentially completely distributive, then $\mathrm{p}(L)$ is completely distributive. Since $L$ is order dense in $\rho(L)$, we conclude from the infinite distributive law that $L^{+-}=L^{-+}=\rho(L)$. We may reformulate this in the following way:

THEOREM 3.7. If $L$ is an essentially completely distributive Zattice, then $p(L)$ is isomorphic with the dual of the latice $F_{c} I_{c}(L)$. The cononical imbedding of $L$ into this lattice is given by

$$
\begin{aligned}
i: L & \rightarrow c_{c}(L) \\
x & \rightarrow\left\{I \in I_{c}(L): x \in I\right\} .
\end{aligned}
$$

This last theorem easily yields the result that when $L$ is the open unit square, $\rho(L)$ is the closed unit square.

\section{References}

[1] Ball, R. "Distributive Cauchy lattices", Algebra Universalis (to appear).

[2] Banaschewski, B. and Bruns, G., "Injective hulls in the category of distributive lattices", J. reine zond angewandte Math. 232 (1968), 102-103.

[3] Birkhoff, G. Lattice Theory, (Amer. Math. Soc. Colloq. Publications, Providence, Rhode Island 1967 ).

[4] Gierz, G., Hofmann, K.H., Keime 1, K., Lawson, J.D., Mislove, M., and Scott, D.S., A Compendium of Continuous Lattices, (Springer Verlag, Berlin, Heidelberg, New York 1980).

[5] Gierz, G. and Stralka, A. R., "Essential extension and congruence extension," Quarterly J. Math. Oxford (2), 35 (1984), 25-36.

[6] Gierz, G. and Stralka, A.R., "The Zariski topology on distributive lattices, (Preprint (1983).

[7] Lawson, J.D., "The duality of continuous posets", Houston J. Math 5 (1979), 357-394.

Department of Mathematics,

University of California,

Riverside,

California 92521 , U.S. A. 